\title{
On some Hölder type trace inequalities for operator weighted geometric mean
}

\author{
S. S. Dragomir
}

Abstract. We obtain some Hölder type trace inequalities for operator weighted geometric mean. Some vector inequalities are also given.

\section{Introduction}

If $\left\{e_{i}\right\}_{i \in I}$ is an orthonormal basis of a Hilbert space $H$, then we say that $A \in \mathcal{B}(H)$ is a trace class provided

$$
\|A\|_{1}:=\sum_{i \in I}\left\langle|A| e_{i}, e_{i}\right\rangle<\infty .
$$

The definition of $\|A\|_{1}$ does not depend on the choice of the orthonormal basis $\left\{e_{i}\right\}_{i \in I}$. We denote by $\mathcal{B}_{1}(H)$ the set of trace class operators in $\mathcal{B}(H)$.

The following properties are also well known:

(i) for any $A \in \mathcal{B}_{1}(H)$ we have

$$
\|A\|_{1}=\left\|A^{*}\right\|_{1}
$$

(ii) $\mathcal{B}_{1}(H)$ is an operator ideal in $\mathcal{B}(H)$, i.e.,

$$
\mathcal{B}(H) \mathcal{B}_{1}(H) \mathcal{B}(H) \subseteq \mathcal{B}_{1}(H) ;
$$

(iii) $\left(\mathcal{B}_{1}(H),\|\cdot\|_{1}\right)$ is a Banach space.

We define the trace of a trace class operator $A \in \mathcal{B}_{1}(H)$ to be

$$
\operatorname{tr}(A):=\sum_{i \in I}\left\langle A e_{i}, e_{i}\right\rangle .
$$

Note that this coincides with the usual definition of the trace if $H$ is finitedimensional. We observe that the series (1.1) converges absolutely and it is independent from the choice of basis.

Received October 11, 2019.

2010 Mathematics Subject Classification. 47A63, 47A30, 26D15, 26D10, 15A60.

Key words and phrases. Young's inequality, Hölder's operator inequality, trace inequalities, arithmetic mean-geometric mean inequality.

https://doi.org/10.12697/ACUTM.2020.24.18 
We collect some properties of the trace:

(i) if $A \in \mathcal{B}_{1}(H)$, then $A^{*} \in \mathcal{B}_{1}(H)$ and

$$
\operatorname{tr}\left(A^{*}\right)=\overline{\operatorname{tr}(A)}
$$

(ii) if $A \in \mathcal{B}_{1}(H)$ and $T \in \mathcal{B}(H)$, then $A T, T A \in \mathcal{B}_{1}(H)$,

$$
\operatorname{tr}(A T)=\operatorname{tr}(T A), \text { and }|\operatorname{tr}(A T)| \leq\|A\|_{1}\|T\| ;
$$

(iii) $\operatorname{tr}(\cdot)$ is a bounded linear functional on $\mathcal{B}_{1}(H)$ with $\|\operatorname{tr}\|=1$;

(iv) $\mathcal{B}_{\text {fin }}(H)$, the space of operators of finite rank, is a dense subspace of $\mathcal{B}_{1}(H)$.

Now, for the finite dimensional case, it is well known that the trace functional is submultiplicative, that is, for positive semidefinite matrices $A$ and $B$ in $M_{n}(\mathbb{C})$,

$$
0 \leq \operatorname{tr}(A B) \leq \operatorname{tr}(A) \operatorname{tr}(B)
$$

Therefore,

$$
0 \leq \operatorname{tr}\left(A^{k}\right) \leq[\operatorname{tr}(A)]^{k},
$$

where $k$ is any positive integer.

In 2000, Yang [22] proved a matrix trace inequality

$$
\operatorname{tr}\left[(A B)^{k}\right] \leq(\operatorname{tr} A)^{k}(\operatorname{tr} B)^{k},
$$

where $A$ and $B$ are positive semidefinite matrices over $\mathbb{C}$ of the same order $n$, and $k$ is any positive integer.

If $(H,\langle\cdot, \cdot\rangle)$ is a separable infinite-dimensional Hilbert space, then the inequality (1.2) is also valid for any positive operators $A, B \in \mathcal{B}_{1}(H)$. This result was obtained by L. Liu in 2007, see [12].

In 2001, Yang et al. [23] improved (1.2) as follows:

$$
\operatorname{tr}\left[(A B)^{m}\right] \leq\left[\operatorname{tr}\left(A^{2 m}\right) \operatorname{tr}\left(B^{2 m}\right)\right]^{1 / 2},
$$

where $A$ and $B$ are positive semidefinite matrices over $\mathbb{C}$ of the same order and $m$ is any positive integer.

In [18] the authors have proved many trace inequalities for sums and products of matrices. For instance, if $A$ and $B$ are positive semidefinite matrices in $M_{n}(\mathbb{C})$, then

$$
\operatorname{tr}\left[(A B)^{k}\right] \leq \min \left\{\|A\|^{k} \operatorname{tr}\left(B^{k}\right),\|B\|^{k} \operatorname{tr}\left(A^{k}\right)\right\}
$$

for any positive integer $k$. Also, if $A, B \in M_{n}(\mathbb{C})$, then for $r \geq 1$ and $p, q>1$ with $1 / p+1 / q=1$ we have the following Young type inequality:

$$
\operatorname{tr}\left(\left|A B^{*}\right|^{r}\right) \leq \operatorname{tr}\left[\left(\frac{|A|^{p}}{p}+\frac{|B|^{q}}{q}\right)^{r}\right] .
$$


Ando [1] proved a strong form of Young's inequality. It was shown that if $A$ and $B$ are in $M_{n}(\mathbb{C})$, then there is a unitary matrix $U$ such that

$$
\left|A B^{*}\right| \leq U\left(\frac{1}{p}|A|^{p}+\frac{1}{q}|B|^{q}\right) U^{*},
$$

where $p, q>1$ with $1 / p+1 / q=1$. This gives immediately the trace inequality

$$
\operatorname{tr}\left(\left|A B^{*}\right|\right) \leq \frac{1}{p} \operatorname{tr}\left(|A|^{p}\right)+\frac{1}{q} \operatorname{tr}\left(|B|^{q}\right) .
$$

This inequality can also be obtained from (1.3) by taking $r=1$.

The following Hölder's type inequality has been proved by Ruskai [16]:

$$
|\operatorname{tr}(A B)| \leq \operatorname{tr}(|A B|) \leq\left[\operatorname{tr}\left(|A|^{p}\right)\right]^{1 / p}\left[\operatorname{tr}\left(|B|^{q}\right)\right]^{1 / q},
$$

where $p, q>1$ with $1 / p+1 / q=1$, and $A, B \in \mathcal{B}(H)$ with $|A|^{p},|B|^{q} \in$ $\mathcal{B}_{1}(H)$.

In particular, for $p=2$ we get the Schwarz inequality

$$
|\operatorname{tr}(A B)| \leq \operatorname{tr}(|A B|) \leq\left[\operatorname{tr}\left(|A|^{2}\right)\right]^{1 / 2}\left[\operatorname{tr}\left(|B|^{2}\right)\right]^{1 / 2}
$$

with $|A|^{2},|B|^{2} \in \mathcal{B}_{1}(H)$.

For the theory of trace functionals and their applications the reader is referred to [20].

For some classical trace inequalities see [4], [6], [14] and [24], which are continuations of the work of Bellman [2]. For related works the reader can refer to [1], [3], [4], [9], [11], [12], [13], [17] and [21].

\section{Some Hölder type trace inequalities}

Assume that $A, B$ are positive invertible operators on a complex Hilbert space $(H,\langle\cdot, \cdot\rangle)$. We use the notation

$$
A \sharp_{\nu} B:=A^{1 / 2}\left(A^{-1 / 2} B A^{-1 / 2}\right)^{\nu} A^{1 / 2}
$$

for the weighted geometric mean. When $\nu=1 / 2$, we write $A \sharp B$ for brevity.

We have the following Hölder type trace inequality.

Theorem 1. If $A, B$ are positive invertible operators, $p, q>1$ with $1 / p+$ $1 / q=1$, and $A^{p}, B^{q} \in \mathcal{B}_{1}(H)$, then $B^{q} \sharp_{1 / p} A^{p} \in \mathcal{B}_{1}(H)$ and

$$
\operatorname{tr}\left(B_{\sharp_{1 / p}} A^{p}\right) \leq\left[\operatorname{tr}\left(A^{p}\right)\right]^{1 / p}\left[\operatorname{tr}\left(B^{q}\right)\right]^{1 / q} .
$$

In particular, if $A^{2}, B^{2} \in \mathcal{B}_{1}(H)$, then $B^{2} \sharp A^{2} \in \mathcal{B}_{1}(H)$ and

$$
\left[\operatorname{tr}\left(B^{2} \sharp A^{2}\right)\right]^{2} \leq \operatorname{tr}\left(A^{2}\right) \operatorname{tr}\left(B^{2}\right) \text {. }
$$


Proof. In [8], the authors obtained the following Hölder's type inequality for the weighted geometric mean:

$$
\left\langle B^{q} \sharp_{1 / p} A^{p} x, x\right\rangle \leq\left\langle A^{p} x, x\right\rangle^{1 / p}\left\langle B^{q} x, x\right\rangle^{1 / q}
$$

for any $x \in H$.

Let $\left\{e_{i}\right\}_{i \in I}$ be an orthonormal basis of $H$. Then by (2.2) and Hölder's inequality we have

$$
\begin{aligned}
\operatorname{tr}\left(B^{q} \sharp_{1 / p} A^{p}\right) & =\sum_{i \in I}\left\langle B^{q} \sharp_{1 / p} A^{p} e_{i}, e_{i}\right\rangle \\
& \leq \sum_{i \in I}\left\langle A^{p} e_{i}, e_{i}\right\rangle^{1 / p}\left\langle B^{q} e_{i}, e_{i}\right\rangle^{1 / q} \\
& \leq\left(\sum_{i \in I}\left[\left\langle A^{p} e_{i}, e_{i}\right\rangle^{1 / p}\right]^{p}\right)^{1 / p}\left(\sum_{i \in I}\left[\left\langle B^{q} e_{i}, e_{i}\right\rangle^{1 / q}\right]^{q}\right)^{1 / q} \\
& =\left(\sum_{i \in I}\left\langle A^{p} e_{i}, e_{i}\right\rangle\right)^{1 / p}\left(\sum_{i \in I}\left\langle B^{q} e_{i}, e_{i}\right\rangle\right)^{1 / q} \\
& =\left[\operatorname{tr}\left(A^{p}\right)\right]^{1 / p}\left[\operatorname{tr}\left(B^{q}\right)\right]^{1 / q}
\end{aligned}
$$

which proves the desired inequality (2.1).

Corollary 1. If $A_{k}, B_{k}$ are positive invertible operators, $p, q>1$ with $1 / p+1 / q=1$, and $A_{k}^{p}, B_{k}^{q} \in \mathcal{B}_{1}(H)$ for $k \in\{1, \ldots, n\}$, then $B_{k}^{q} \sharp_{1 / p} A_{k}^{p} \in$ $\mathcal{B}_{1}(H)$ for $k \in\{1, \ldots, n\}$, and for any $p_{k} \geq 0, k \in\{1, \ldots, n\}$, we have

$$
\operatorname{tr}\left(\sum_{k=1}^{n} p_{k} B_{k}^{q} \sharp_{1 / p} A_{k}^{p}\right) \leq\left(\operatorname{tr}\left(\sum_{k=1}^{n} p_{k} A_{k}^{p}\right)\right)^{1 / p}\left(\operatorname{tr}\left(\sum_{k=1}^{n} p_{k} B_{k}^{q}\right)\right)^{1 / q} .
$$

In particular, if $A_{k}^{2}, B_{k}^{2} \in \mathcal{B}_{1}(H)$ for $k \in\{1, \ldots, n\}$, then $B_{k}^{2} \sharp A_{k}^{2} \in \mathcal{B}_{1}(H)$ for $k \in\{1, \ldots, n\}$, and for any $p_{k} \geq 0, k \in\{1, \ldots, n\}$, we have

$$
\left[\operatorname{tr}\left(\sum_{k=1}^{n} p_{k} B_{k}^{2 \sharp} A_{k}^{2}\right)\right]^{2} \leq \operatorname{tr}\left(\sum_{k=1}^{n} p_{k} A_{k}^{2}\right) \operatorname{tr}\left(\sum_{k=1}^{n} p_{k} B_{k}^{2}\right) .
$$

Proof. Using Hölder's weighted discrete inequality, we have

$$
\begin{aligned}
\operatorname{tr}\left(\sum_{k=1}^{n} p_{k} B_{k}^{q_{1}}{ }_{1 / p} A_{k}^{p}\right) & =\sum_{k=1}^{n} p_{k} \operatorname{tr}\left(B_{k}^{q} \sharp_{1 / p} A_{k}^{p}\right) \leq \sum_{k=1}^{n} p_{k}\left[\operatorname{tr}\left(A_{k}^{p}\right)\right]^{1 / p}\left[\operatorname{tr}\left(B_{k}^{q}\right)\right]^{1 / q} \\
& \leq\left(\sum_{k=1}^{n} p_{k}\left(\left[\operatorname{tr}\left(A_{k}^{p}\right)\right]^{1 / p}\right)^{p}\right)^{1 / p}\left(\sum_{k=1}^{n} p_{k}\left(\left[\operatorname{tr}\left(B_{k}^{q}\right)\right]^{1 / q}\right)^{q}\right)^{1 / q}
\end{aligned}
$$




$$
\begin{aligned}
& =\left(\sum_{k=1}^{n} p_{k} \operatorname{tr}\left(A_{k}^{p}\right)\right)^{1 / p}\left(\sum_{k=1}^{n} p_{k} \operatorname{tr}\left(B_{k}^{q}\right)\right)^{1 / q} \\
& =\left(\operatorname{tr}\left(\sum_{k=1}^{n} p_{k} A_{k}^{p}\right)\right)^{1 / p}\left(\operatorname{tr}\left(\sum_{k=1}^{n} p_{k} B_{k}^{q}\right)\right)^{1 / q}
\end{aligned}
$$

and the inequality (2.3) is proved.

Theorem 2. If $A, B$ are positive invertible operators, $p, q>1$ with $1 / p+$ $1 / q=1$, and $C \in \mathcal{B}_{1}(H), C \geq 0$, then $C A^{p}, C B^{q}, C\left(B_{\sharp_{1 / p}}^{A^{p}}\right) \in \mathcal{B}_{1}(H)$ and

$$
\operatorname{tr}\left(C\left(B^{q} \sharp_{1 / p} A^{p}\right)\right) \leq\left[\operatorname{tr}\left(C A^{p}\right)\right]^{1 / p}\left[\operatorname{tr}\left(C B^{q}\right)\right]^{1 / q} .
$$

In particular, if $C \in \mathcal{B}_{1}(H)$, then $C A^{2}, C B^{2}, C\left(B^{2} \sharp A^{2}\right) \in \mathcal{B}_{1}(H)$ and

$$
\left[\operatorname{tr}\left(C\left(B^{2} \sharp A^{2}\right)\right)\right]^{2} \leq \operatorname{tr}\left(C A^{2}\right) \operatorname{tr}\left(C B^{2}\right) .
$$

Proof. From the inequality (2.2) we have

$$
\left\langle B_{\sharp_{1 / p}} A^{p} C^{1 / 2} x, C^{1 / 2} x\right\rangle \leq\left\langle A^{p} C^{1 / 2} x, C^{1 / 2} x\right\rangle^{1 / p}\left\langle B^{q} C^{1 / 2} x, C^{1 / 2} x\right\rangle^{1 / q}
$$

for any $x \in H$, which is equivalent to

$$
\left\langle C^{1 / 2} B_{\sharp_{1} / p} A^{p} C^{1 / 2} x, x\right\rangle \leq\left\langle C^{1 / 2} A^{p} C^{1 / 2} x, x\right\rangle^{1 / p}\left\langle C^{1 / 2} B^{q} C^{1 / 2} x, x\right\rangle^{1 / q}
$$

for any $x \in H$.

Let $\left\{e_{i}\right\}_{i \in I}$ be an orthonormal basis of $H$. Then by (2.5) and Hölder's inequality we have

$$
\begin{aligned}
\operatorname{tr} & \left(C\left(B_{\sharp_{1 / p}}^{q^{p}} A^{p}\right)\right) \\
& =\operatorname{tr}\left(C^{1 / 2}\left(B^{q} \sharp_{1 / p} A^{p}\right) C^{1 / 2}\right)=\sum_{i \in I}\left\langle C^{1 / 2}\left(B^{q} \sharp_{1 / p} A^{p}\right) C^{1 / 2} e_{i}, e_{i}\right\rangle \\
& \leq \sum_{i \in I}\left\langle C^{1 / 2} A^{p} C^{1 / 2} e_{i}, e_{i}\right\rangle^{1 / p}\left\langle C^{1 / 2} B^{q} C^{1 / 2} e_{i}, e_{i}\right\rangle^{1 / q} \\
& \leq\left(\sum_{i \in I}\left[\left\langle C^{1 / 2} A^{p} C^{1 / 2} e_{i}, e_{i}\right\rangle^{1 / p}\right]^{p}\right)^{1 / p}\left(\sum_{i \in I}\left[\left\langle C^{1 / 2} B^{q} C^{1 / 2} e_{i}, e_{i}\right\rangle^{1 / q}\right]^{q}\right)^{1 / q} \\
& =\left(\sum_{i \in I}\left\langle C^{1 / 2} A^{p} C^{1 / 2} e_{i}, e_{i}\right\rangle\right)^{1 / p}\left(\sum_{i \in I}\left\langle C^{1 / 2} B^{q} C^{1 / 2} e_{i}, e_{i}\right\rangle\right)^{1 / q} \\
& =\left[\operatorname{tr}\left(C^{1 / 2} A^{p} C^{1 / 2}\right)\right]^{1 / p}\left[\operatorname{tr}\left(C^{1 / 2} B^{q} C^{1 / 2}\right)\right]^{1 / q}=\left[\operatorname{tr}\left(C A^{p}\right)\right]^{1 / p}\left[\operatorname{tr}\left(C B^{q}\right)\right]^{1 / q},
\end{aligned}
$$

which proves the desired result (2.4). 
Corollary 2. If $A_{k}, B_{k}$ are positive invertible operators, $p, q>1$ with $1 / p+1 / q=1$, and $C_{k} \in \mathcal{B}_{1}(H), C_{k} \geq 0$ for $k \in\{1, \ldots, n\}$, then $C_{k} A_{k}^{p}$, $C_{k} B_{k}^{q}, C_{k}\left(B_{k}^{q_{1} / p} A_{k}^{p}\right) \in \mathcal{B}_{1}(H)$ for $k \in\{1, \ldots, n\}$ and we have

$$
\operatorname{tr}\left(\sum_{k=1}^{n} C_{k}\left(B_{k}^{q_{1} / p} A_{k}^{p}\right)\right) \leq\left(\operatorname{tr}\left(\sum_{k=1}^{n} C_{k} A_{k}^{p}\right)\right)^{1 / p}\left(\operatorname{tr}\left(\sum_{k=1}^{n} C_{k} B_{k}^{q}\right)\right)^{1 / q} .
$$

In particular, $C_{k} A_{k}^{2}, C_{k} B_{k}^{2}, C_{k}\left(B_{k}^{2 \sharp} A_{k}^{2}\right) \in \mathcal{B}_{1}(H)$ for $k \in\{1, \ldots, n\}$ and

$$
\left[\operatorname{tr}\left(\sum_{k=1}^{n} C_{k}\left(B_{k}^{2} \sharp A_{k}^{2}\right)\right)\right]^{2} \leq \operatorname{tr}\left(\sum_{k=1}^{n} C_{k} A_{k}^{2}\right) \operatorname{tr}\left(\sum_{k=1}^{n} C_{k} B_{k}^{2}\right) .
$$

The proof follows by (2.4) making use of a similar argument to the one in the proof of Corollary 1.

\section{Some reverse vector inequalities}

We have the following reverse of Hölder's vector inequality for operators.

Theorem 3. Let $A$ and $B$ be two positive invertible operators, $p, q>1$ with $1 / p+1 / q=1$ and let $m, M>0$ be such that

$$
m^{p} B^{q} \leq A^{p} \leq M^{p} B^{q} .
$$

Then

$$
\left\langle A^{p} x, x\right\rangle^{1 / p}\left\langle B^{q} x, x\right\rangle^{1 / q} \leq \exp \left[\frac{1}{2 p q}\left(\left(\frac{M}{m}\right)^{p}-1\right)^{2}\right]\left\langle B_{\sharp_{1} / p} A^{p} x, x\right\rangle
$$

for any $x \in H$.

Proof. In [7] we proved the following double inequality that provides a refinement and a reverse of the arithmetic mean-geometric mean inequality:

$$
\begin{aligned}
\exp \left[\frac{1}{2} \nu(1-\nu)\left(1-\frac{\min \{a, b\}}{\max \{a, b\}}\right)^{2}\right] \leq \frac{(1-\nu) a+\nu b}{a^{1-\nu} b^{\nu}} \\
\leq \exp \left[\frac{1}{2} \nu(1-\nu)\left(\frac{\max \{a, b\}}{\min \{a, b\}}-1\right)^{2}\right]
\end{aligned}
$$

for any $a, b>0$ and $\nu \in[0,1]$.

If $a, b \in[t, T] \subset(0, \infty)$ and since

we have

$$
0<\frac{\max \{a, b\}}{\min \{a, b\}}-1 \leq \frac{T}{t}-1
$$

$$
\left(\frac{\max \{a, b\}}{\min \{a, b\}}-1\right)^{2} \leq\left(\frac{T}{t}-1\right)^{2}
$$


Therefore, by (3.3) we get

$$
(1-\nu) a+\nu b \leq a^{1-\nu} b^{\nu} \exp \left[\frac{1}{2} \nu(1-\nu)\left(\frac{T}{t}-1\right)^{2}\right],
$$

for any $a, b \in[t, T]$ and $\nu \in(0,1)$.

Now, if $C$ is an operator with $t I \leq C \leq T I$, then for $p>1$ we have $t^{p} I \leq C^{p} \leq T^{p} I$. Using the functional calculus, we get from (3.4) for $\nu=\frac{1}{p}$ that

$$
\left(1-\frac{1}{p}\right) d+\frac{1}{p} C^{p} \leq \exp \left[\frac{1}{2 p q}\left(\left(\frac{T}{t}\right)^{p}-1\right)^{2}\right] d^{1-\frac{1}{p}} C,
$$

namely, the vector inequality

$$
\left(1-\frac{1}{p}\right) d+\frac{1}{p}\left\langle C^{p} y, y\right\rangle \leq \exp \left[\frac{1}{2 p q}\left(\left(\frac{T}{t}\right)^{p}-1\right)^{2}\right] d^{1-\frac{1}{p}}\langle C y, y\rangle,
$$

for any $y \in H,\|y\|=1$ and $d \in\left[t^{p}, T^{p}\right]$.

Since $d=\left\langle C^{p} y, y\right\rangle \in\left[t^{p}, T^{p}\right]$ for any $y \in H,\|y\|=1$, and hence by (3.5) we have

$$
\left(1-\frac{1}{p}\right)\left\langle C^{p} y, y\right\rangle+\frac{1}{p}\left\langle C^{p} y, y\right\rangle \leq \exp \left[\frac{1}{2 p q}\left(\left(\frac{T}{t}\right)^{p}-1\right)^{2}\right]\left\langle C^{p} y, y\right\rangle^{1-\frac{1}{p}}\langle C y, y\rangle,
$$

which is equivalent to

$$
\left\langle C^{p} y, y\right\rangle \leq \exp \left[\frac{1}{2 p q}\left(\left(\frac{T}{t}\right)^{p}-1\right)^{2}\right]\left\langle C^{p} y, y\right\rangle^{1-\frac{1}{p}}\langle C y, y\rangle,
$$

and by division with $\left\langle C^{p} y, y\right\rangle^{1-\frac{1}{p}}>0, y \in H,\|y\|=1$, to

$$
\left\langle C^{p} y, y\right\rangle^{1 / p} \leq \exp \left[\frac{1}{2 p q}\left(\left(\frac{T}{t}\right)^{p}-1\right)^{2}\right]\langle C y, y\rangle .
$$

If $z \in H$ with $z \neq 0$, then by taking $y=\frac{z}{\|z\|}$ in (3.6) we get

$$
\left\langle C^{p} z, z\right\rangle^{1 / p}\langle z, z\rangle^{1 / q} \leq \exp \left[\frac{1}{2 p q}\left(\left(\frac{T}{t}\right)^{p}-1\right)^{2}\right]\langle C z, z\rangle,
$$

for any $z \in H$.

Now, from (3.1) by multiplying both sides with $B^{-\frac{q}{2}}$, we have $m^{p} I \leq B^{-\frac{q}{2}} A^{p} B^{-\frac{q}{2}} \leq M^{p} I$, and by taking the power $1 / p$ we get $m I \leq$ $\left(B^{-\frac{q}{2}} A^{p} B^{-\frac{q}{2}}\right)^{\frac{1}{p}} \leq M I$. 
Writing the inequality (3.7) for $C=\left(B^{-\frac{q}{2}} A^{p} B^{-\frac{q}{2}}\right)^{\frac{1}{p}}, t=m, T=M$ and $z=B^{\frac{q}{2}} x$, with $x \in H$, we have

$$
\begin{aligned}
& \left\langle B^{-\frac{q}{2}} A^{p} B^{-\frac{q}{2}} B^{\frac{q}{2}} x, B^{\frac{q}{2}} x\right\rangle^{1 / p}\left\langle B^{\frac{q}{2}} x, B^{\frac{q}{2}} x\right\rangle^{1 / q} \\
& \leq \exp \left[\frac{1}{2 p q}\left(\left(\frac{M}{m}\right)^{p}-1\right)^{2}\right]\left\langle\left(B^{-\frac{q}{2}} A^{p} B^{-\frac{q}{2}}\right)^{\frac{1}{p}} B^{\frac{q}{2}} x, B^{\frac{q}{2}} x\right\rangle,
\end{aligned}
$$

namely

$$
\begin{aligned}
& \left\langle A^{p} x, x\right\rangle^{1 / p}\left\langle B^{q} x, x\right\rangle^{1 / q} \\
& \leq \exp \left[\frac{1}{2 p q}\left(\left(\frac{M}{m}\right)^{p}-1\right)^{2}\right]\left\langle B^{\frac{q}{2}}\left(B^{-\frac{q}{2}} A^{p} B^{-\frac{q}{2}}\right)^{\frac{1}{p}} B^{\frac{q}{2}} x, x\right\rangle,
\end{aligned}
$$

for any $x \in H$. The inequality (3.2) is proved.

Remark 1. We observe, for two positive invertible operators $A$ and $B$, that the condition (3.1) is equivalent to condition

$$
m I \leq\left(B^{-\frac{q}{2}} A^{p} B^{-\frac{q}{2}}\right)^{\frac{1}{p}} \leq M I .
$$

If we assume that $r B^{q} \leq A^{p} \leq R B^{q}$, then by (3.2) we have the inequality

$$
\left\langle A^{p} x, x\right\rangle^{1 / p}\left\langle B^{q} x, x\right\rangle^{1 / q} \leq \exp \left[\frac{1}{2 p q}\left(\frac{R}{r}-1\right)^{2}\right]\left\langle B^{q_{\sharp}}{ }_{1 / p} A^{p} x, x\right\rangle
$$

for any $x \in H$.

The following particular case is related to Schwarz's trace inequality.

Corollary 3. Let $A$ and $B$ be two positive invertible operators and let $m$, $M>0$ be such that

$$
m I \leq\left(B^{-1} A^{2} B^{-1}\right)^{\frac{1}{2}} \leq M I
$$

Then we have

$$
\left\langle A^{2} x, x\right\rangle^{1 / 2}\left\langle B^{2} x, x\right\rangle^{1 / 2} \leq \exp \left[\frac{1}{8}\left(\left(\frac{M}{m}\right)^{2}-1\right)^{2}\right]\left\langle A^{2} \sharp B^{2} x, x\right\rangle
$$

for any $x \in H$.

Under more suitable conditions for the operators involved, we have the following result.

Corollary 4. Assume that $A$ and $B$ satisfy the conditions

$$
m_{1} I \leq A \leq M_{1} I, m_{2} I \leq B \leq M_{2} I
$$


for some $0<m_{1}<M_{1}$ and $0<m_{2}<M_{2}$. Then we have

$$
\left\langle A^{p} x, x\right\rangle^{1 / p}\left\langle B^{q} x, x\right\rangle^{1 / q} \leq \exp \left[\frac{1}{2 p q}\left(\left(\frac{M_{1}}{m_{1}}\right)^{p}\left(\frac{M_{2}}{m_{2}}\right)^{q}-1\right)^{2}\right]\left\langle B^{q_{\sharp} / p} A^{p} x, x\right\rangle,
$$

for any $x \in H$.

In particular, we have

$$
\left\langle A^{2} x, x\right\rangle^{1 / 2}\left\langle B^{2} x, x\right\rangle^{1 / 2} \leq \exp \left[\frac{1}{8}\left(\left(\frac{M_{1} M_{2}}{m_{1} m_{2}}\right)^{2}-1\right)^{2}\right]\left\langle A^{2} \sharp B^{2} x, x\right\rangle,
$$

for any $x \in H$.

\section{References}

[1] T. Ando, Matrix Young inequalities, Oper. Theory Adv. Appl. 75 (1995), 33-38.

[2] R. Bellman, Some inequalities for positive definite matrices, in: General Inequalities 2 (Proc. Second Internat. Conf., Oberwolfach, 1978), Birkhäuser, Basel-Boston, 1980, pp. 89-90.

[3] E. V. Belmega, M. Jungers, and S. Lasaulce, A generalization of a trace inequality for positive definite matrices, Aust. J. Math. Anal. Appl. 7(2) (2010), Art. 26, 5 pp.

[4] D. Chang, A matrix trace inequality for products of Hermitian matrices, J. Math. Anal. Appl. 237 (1999), 721-725.

[5] L. Chen and C. Wong, Inequalities for singular values and traces, Linear Algebra Appl. 171 (1992), 109-120.

[6] I. D. Coop, On matrix trace inequalities and related topics for products of Hermitian matrix, J. Math. Anal. Appl. 188 (1994), 999-1001.

[7] S. S. Dragomir, A note on new refinements and reverses of Young's inequality, Preprint RGMIA Res. Rep. Coll. , 18 (2015), Art. 131. Online http://rgmia.org/papers/v18/v18a131.pdf.

[8] M. Fuji, S. Izumino, R. Nakamoto, and Y. Seo, Operator inequalities related to Cauchy-Schwarz and Hölder-McCarthy inequalities, Nihonkai Math. J. 8 (1997), 117122.

[9] S. Furuichi and M. Lin, Refinements of the trace inequality of Belmega, Lasaulce and Debbah, Aust. J. Math. Anal. Appl. 7(2) (2010), Art. 23, 4 pp.

[10] W. Greub and W. Rheinboldt, On a generalisation of an inequality of L.V. Kantorovich, Proc. Amer. Math. Soc. 10 (1959), 407-415.

[11] H. D. Lee, On some matrix inequalities, Korean J. Math. 16(4) (2008), 565-571.

[12] L. Liu, A trace class operator inequality, J. Math. Anal. Appl. 328 (2007) 1484-1486.

[13] S. Manjegani, Hölder and Young inequalities for the trace of operators, Positivity 11 (2007), 239-250.

[14] H. Neudecker, A matrix trace inequality, J. Math. Anal. Appl. 166 (1992) 302-303.

[15] J. Pečarić, T. Furuta, J. Mićić Hot, and Y. Seo, Mond-Pečarić Method in Operator Inequalities. Inequalities for Bounded Selfadjoint Operators on a Hilbert Space, Element, Zagreb, 2005.

[16] M. B. Ruskai, Inequalities for traces on von Neumann algebras, Commun. Math. Phys. 26 (1972), 280-289.

[17] K. Shebrawi and H. Albadawi, Operator norm inequalities of Minkowski type, J. Inequal. Pure Appl. Math. 9(1) (2008), 1-10. 
[18] K. Shebrawi and H. Albadawi, Trace inequalities for matrices, Bull. Aust. Math. Soc. 87 (2013), 139-148.

[19] O. Shisha and B. Mond, Bounds on differences of means, in: Inequalities (Proc. Sympos. Wright-Patterson Air Force Base, Ohio, 1965), Academic Press, New York, 1967, pp. 293-308.

[20] B. Simon, Trace Ideals and Their Applications, Cambridge University Press, Cambridge, 1979.

[21] Z. Ulukök and R. Türkmen, On some matrix trace inequalities, J. Inequal. Appl. 2010, Art. ID 201486, 8 pp.

[22] X. Yang, A matrix trace inequality, J. Math. Anal. Appl. 250 (2000) 372-374.

[23] X. M. Yang, X. Q. Yang, and K. L. Teo, A matrix trace inequality, J. Math. Anal. Appl. 263 (2001), 327-331.

[24] Y. Yang, A matrix trace inequality, J. Math. Anal. Appl. 133 (1988) 573-574.

Mathematics, College of Engineering \& Science, Victoria University, PO Box 14428, Melbourne City, MC 8001, Australia; DST-NRF Centre of ExcelLence, in the Mathematical and Statistical Sciences, School of Computational \& Applied Mathematics, University of the Witwatersrand, Private Bag 3, JoHANNESBURg 2050, South AfRicA

E-mail address: sever.dragomir@vu.edu.au

$U R L$ : http://rgmia.org/dragomir 